DOI: $10.5216 /$ cab.v13i3.4815

\title{
EFEITO DE DIFERENTES DOSES DE CLOPROSTENOL SÓDICO NO PERÍODO PÓS-PARTO DE VACAS DE CORTE
}

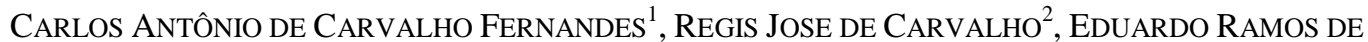 \\ Oliveira $^{3}$, JoÃo Henrique Moreira Viana ${ }^{4}$, Miller Pereira Palhão ${ }^{1}$, Marilu Martins Gioso $^{1}$ \\ ${ }^{1}$ Professores Doutores da Universidade José do Rosário Vellano, Alfenas, MG, Brasil - cacf@ biotran.com.br. \\ ${ }^{2}$ Mestrando Reprodução animal - Universidade José do Rosário Vellano, Alfenas, MG, Brasil \\ ${ }^{3}$ Biotran - Biotecnologia e Treinamento em Reprodução Animal, Alfenas, MG, Brasil. \\ ${ }^{4}$ Pesquisador Doutor da EMBRAPA - Centro Nacional de Pesquisa de Gado de Leite, Juiz de Fora, MG, Brasil.
}

\section{RESUMO}

O restabelecimento da atividade reprodutiva pós-parto é dependente de dois processos fisiológicos, a involução uterina e o restabelecimento da atividade luteal cíclica. Problemas ou atrasos na involução uterina podem afetar diretamente a atividade ovariana pós-parto. As prostaglandinas $\mathrm{F} 2 \alpha(\mathrm{PGF} 2 \alpha)$ exercem uma importante função no processo de involução uterina. Entretanto, a utilização dos análogos sintéticos da PGF2 $\alpha$ para estimular involução uterina em bovinos tem sido pequena. O objetivo deste estudo foi avaliar o efeito de duas doses de uma mistura racêmica de cloprostenol (D+LCloprostenol), aplicadas no pós-parto imediato, sobre o desempenho reprodutivo de vacas mestiças de corte. Vacas de corte com parto normal foram divididas aleatoriamente em três grupos: $\mathrm{G} 1(\mathrm{n}=144)$, grupo controle; G2 $(\mathrm{n}=145), 0.530 \mathrm{mg}$ de D+L-Cloprostenol, aplicados IM de três a cinco dias após o parto, e G3 $(\mathrm{n}=145), 1.060 \mathrm{mg}$ de D+L-Cloprostenol, no mesmo protocolo de G2. Foram analisados os serviços por concepção $\left(\chi^{2}\right)$, dias do parto à primeira inseminação e período de serviço. Não foram observadas diferenças no número de serviços por concepção nos três grupos $(\mathrm{P}>0.05)$. A média de dias do parto à primeira inseminação foi de $88,77 \pm 23,64^{\mathrm{a}} ; 77,59 \pm 26,95^{\mathrm{b}}$ e 76,22 $\pm 26,28^{\mathrm{b}}$, e o intervalo parto-concepção foi de $97.34 \pm 26.54^{\mathrm{a}} ; 86,38 \pm 28,81^{\mathrm{b}} ; 85,23 \pm 30,12^{\mathrm{b}}$, para os grupos 1,2 e 3 , respectivamente $(\mathrm{P}<0.05)$. $\mathrm{O}$ tratamento, independente da dose de cloprostenol, antecipou o reinicio da atividade reprodutiva em mais de 10 dias. A aplicação de cloprostenol sódico no pós-parto pode melhorar a eficiência reprodutiva de vacas de corte. Não existem diferenças entre as duas doses comparadas.

PALAVRAS-CHAVE: bovino; involução uterina; prostaglandinas.

\section{EFFECT OF DIFFERENT DOSES OF SODIC CLOPROSTENOL AT POST-PARTUM PERIOD OF BEEF COWS}

\section{ABSTRACT}

Reestablishment of reproductive activity after parturition is dependent on two physiological processes, uterine involution and reestablishment of the ovarian luteal cyclic activity. Impaired or delayed uterine involution can affect ovarian activity. Prostaglandin F2 $\alpha$ (PGF2 $\alpha$ ) has an important function in uterine involution. The use of PGF2 $\alpha$ synthetic analogous in bovine postpartum, however, has been limited. The aim of this study was to compare the effect of two different doses of a racemic cloprostenol mixture (D+L-Cloprostenol) given in the postpartum period, on reproductive performance of crossbred beef cows. Beef cows with normal parturition were randomly distributed into three groups: $\mathrm{G} 1(\mathrm{n}=144)$, Control Group; G2 ( $\mathrm{n}=145), 0.530 \mathrm{mg}$ of $\mathrm{D}+\mathrm{L}-$ Cloprostenol, given IM at three to five days after parturition, and the G3 $(\mathrm{n}=145), 1.060 \mathrm{mg}$ of $\mathrm{D}$ Cloprostenol, in the same schedule of group 2. The following parameters evaluated were: services per conception $\left(\chi^{2}\right)$, days from parturition to first estrus, and open days (Tukey test). There was no difference in body score condition among groups at parturition or during postpartum period $(\mathrm{P}>0.05)$. There was also no difference 
in the number of services per conception. The average number of days from parturition to first estrus was $88.77 \pm$ $23.64^{\mathrm{a}} ; 77.59 \pm 26.95^{\mathrm{b}}$ and $76.22 \pm 26.28^{\mathrm{b}}$, and the average number of open days open was $97.34 \pm 26.54^{\mathrm{a}}$, $86.38 \pm 28.81^{\mathrm{b}}$ and $85.23 \pm 30.12^{\mathrm{b}}$ for groups 1,2 and 3 , respectively $(\mathrm{P}<0.05)$. Regardless cloprostenol doses, the

KEYWORDS: bovine; prostaglandins; uterine involution.

\section{INTRODUÇÃO}

O período que vai do parto ao reinício da atividade reprodutiva depende, basicamente, de dois processos fisiológicos, relacionando-se com o período de involução uterina e também com o tempo necessário para o reinicio da atividade ovariana cíclica, sendo esse período definido como puerpério (FERREIRA et al., 1999). Para a vaca, puerpério tem sido definido como o período que vai do parto até o aparecimento do primeiro estro, no qual nova gestação pode ser estabelecida, o que implica completa involução uterina e retorno da atividade endócrina, com plena reativação e sincronia do eixo hipotálamo-hipófise-ovário, permitindo crescimento folicular, estro, ovulação, concepção, desenvolvimento do corpo lúteo e gestação (MARQUES JÚNIOR, 1993).

Não existe sincronia entre o inicio da atividade ovariana, que se reinicia e pode estar completa em torno de duas a três semanas após o parto, e a involução uterina, que parece estar completa em torno de 45 dias (MARQUES JÚNIOR, 1993). Alguns autores, porém, relatam que, para o retorno à atividade reprodutiva, é importante uma involução uterina rápida. Segundo SHELDON et al. (2000), existe uma grande correlação entre a involução uterina e o retorno à atividade ovariana pós-parto. Esses autores afirmam que o retardo na involução uterina pode atrasar a presença de atividade funcional nos ovários, retardando o início do primeiro cio pós-parto.

Segundo MARQUES JÚNIOR (1993), o período puerperal é dos mais importantes no contexto do manejo, por representar o período de maior vulnerabilidade do animal a problemas que afetam a fertilidade futura e a eficiência reprodutiva. A vaca tem um tipo de involução do útero bastante complexa, em parte, devido ao tipo de placenta presente nessa espécie. Após a parição, os cotilédones fetais se separam das carúnculas maternas. As carúnculas e o restante da parede uterina sofrem um processo semelhante a um processo inflamatório. A massa uterina, logo após o parto normal, pesa aproximadamente $10 \mathrm{~kg}$, caindo treatments anticipated the beginning of reproductive activity in more than 10 days. The treatment with sodic cloprostenol in the post-partum period of zebu beef cows is an alternative to reduce anestrous postpartum and open days.

para $0,7-0,8 \mathrm{~kg}$ até a sexta semana após o parto, quando ocorre uma involução total. Assim, no período logo após o parto, observa-se uma mudança na massa do tecido uterino de mais de 10 vezes o seu tamanho (KASK et al., 1999).

$O$ processo de involução envolve modificações na camada interna de revestimento e também a atividade contrátil do miométrio, para expulsão do conteúdo remanescente pós-parto e também redução do tamanho do útero. A involução uterina pode ser comparada a um processo de reação inflamatória (SHELDON et al., 2000). CHAGAS et al. (1998), num trabalho de avaliação histológica do útero de vacas no pós-parto, observaram que aquelas que apresentaram achados mais evidentes de processo inflamatório tiveram involução mais rápida.

Como nos casos de inflamação, na involução uterina, as prostaglandinas exercem importante função (BENCHARIF et al., 2000). Além de acelerar o processo de involução, a prostaglandina $\mathrm{F} 2 \alpha$ (PGF2 $\alpha$ ) estimula a atividade da camada muscular uterina (miométrio) após o parto. Essa substância é normalmente produzida pelo útero sendo responsável pela correta involução uterina pós-parto, em tempo normal. TANIKAWA et al. (2005) indicam que as prostaglandinas, além de importante papel no pósparto imediato, atuam também após a completa involução uterina, participando de vários eventos fisiológicos.

Os análogos sintéticos da PGF2 $\alpha$ têm sido amplamente utilizados para controlar a atividade funcional do corpo lúteo, porém sua aplicação no pós-parto em bovinos tem sido pouco empregada em nosso país (FERNANDES \& FIGUEIREDO, 2007), embora seja rotina em outros (BENCHARIF et al., 2000). ALBUQUERQUE et al. (1997) citam efeito benéfico na involução uterina de vacas tratadas com o mesmo análogo sintético. Esses autores mostram resultados na melhoria do desempenho reprodutivo quando o cloprostenol foi aplicado entre 12 e 18 dias após o parto.

FERNANDES (1999), num trabalho com animais com retenção de placenta, constatou efeitos benéficos da aplicação do cloprostenol sódico. As vacas tratadas exibiram involução uterina mais 
rápida e também um menor intervalo parto-primeiro estro, em relação àquelas apresentando a mesma condição, porém não tratadas com este fármaco. ZAIEM et al. (1997) mostraram que vacas que receberam uma aplicação de análogo sintético da prostaglandina no pós-parto apresentaram involução uterina mais rápida que animais do grupo controle.

A partir dos trabalhos inicialmente publicados por FERNANDES et al. (2002ab), a utilização do cloprostenol sódico no pós-parto de vacas leiteiras se tornou uma realidade. Esses trabalhos mostraram que, além de acelerar a involução uterina, reduziram-se a incidência de infecções, o número de serviços por concepção e o período de serviços. Vários outros trabalhos, em diferentes sistemas de manejo, corroboraram posteriormente esses achados (FIGUEIREDO et al., 2000; ZANCHET, 2005).

A utilização de análogos de prostaglandina no pós-parto de gado de corte também se mostrou ser um protocolo muito eficiente em duas aplicações (FERNANDES et al. 2005). Além de simples, apresenta uma ótima relação custo-benefício. Esse tipo de protocolo, no entanto, tem encontrado resistência para utilização em gado de corte, pelas dificuldades de manejo referentes a duas aplicações. Uma alternativa para tornar viável a utilização desse protocolo num esquema de manejo normalmente existente em gado de corte seria a utilização de doses maiores numa única aplicação.

O objetivo deste trabalho foi avaliar a aplicação de duas doses de cloprostenol sódico (análogo da prostaglandinaF2 $\alpha$ ) no pós-parto imediato sobre o desempenho reprodutivo de vacas de corte.

\section{MATERIAL E MÉTODOS}

O trabalho foi conduzido num período de seis meses nas dependências de uma fazenda de gado de corte, localizada no sudoeste do Estado de Minas Gerais. Essa propriedade possuía apenas inseminação artificial como forma de cobertura das vacas. O rebanho utilizado era composto basicamente por animais mestiços da raça Nelore com raças europeias. A propriedade adota o sistema de estação de monta de 90 dias para a inseminação das fêmeas. Existem três núcleos (rebanhos, funcionários e infra-estrutura) que funcionam como propriedades distintas, nos quais a mão-de-obra e manejo dos animais são independentes.

Para se evitar influencia de outras variáveis, como manejo, aspectos climáticos e nutricionais, o experimento foi conduzido, em cada um dos núcleos, avaliando-se os diferentes grupos de tratamento simultaneamente.

Foram utilizadas 461 vacas de corte, apresentando parto normal (sem qualquer intervenção ou registro de problemas). Após o parto, os animais foram divididos aleatoriamente em três grupos de tratamento conforme descrito na Tabela 1.

Um funcionário da propriedade, em cada um dos núcleos, foi treinado para anotações (tipo de tratamento, data do parto, escore corporal e outros), procedimentos adicionais e aplicação dos produtos. Além de receber uma planilha para anotações, com os tratamentos pré-determinados, nos quais as fêmeas foram distribuídas após o parto. Um grupo de vacas foi utilizado como controle, ou seja, não tratados, e as variáveis foram comparadas com os outros grupos. As fêmeas do grupo controle foram tratadas com salina.

Tabela 1: Esquema de tratamento dos animais após o parto

\begin{tabular}{lll}
\hline Grupo & $\mathrm{N}$ & Esquema de tratamento \\
\hline Grupo 1 - controle & 152 & $2 \mathrm{~mL}$ de salina intramuscular - Grupo Controle \\
Grupo 2 & 151 & $0,530 \mathrm{mg}$ de cloprostenol via intramuscular, no dia do parto ou dia seguinte \\
Grupo 3 & 158 & $1,060 \mathrm{mg}$ de cloprostenol via intramuscular, no dia do parto ou dia seguinte \\
\hline
\end{tabular}

Foi avaliado o escore de condição corporal, em uma escala de 1 a 5 , conforme citado por FERNANDES et al. (2002a), no momento da aplicação e entre 30 e 45 dias após o parto. A comparação da média dessas variáveis foi utilizada para se definir o padrão de homogeneidade referente aos aspectos nutricionais entre os grupos.

Para os tratamentos, todos via intramuscular na região do glúteo, foram utilizadas seringas de $3 \mathrm{~mL}$ e agulhas $40 \times 8$, ambas descartáveis. Durante o período experimental, a propriedade foi visitada regularmente, em intervalos mensais, para realização de exames ginecológicos e coleta de dados.

Em relação ao desempenho reprodutivo, foram avaliadas e comparadas as seguintes variáveis: intervalo parto-primeira IA, número de serviços/concepção e período de serviços. Esses dados foram submetidos à análise de variância (Anova) e, quando houve diferença, as médias foram comparadas pelo teste de Tukey. O escore de 
condição corporal (ECC) logo após o parto e 30-45 dias pós-parto foi avaliado pela prova não paramétrica de Wilcoxon. Para as análises estatísticas foi utilizado o programa SAEG.

\section{RESULTADOS E DISCUSSÃO}

O escore de condição corporal médio dos animais não diferiu entre os núcleos avaliados nem nos diferentes tratamentos $(\mathrm{P}>0,05)$, considerando um mesmo período de análise (pré ou pós-parto). Foram observadas diferenças de escore de condição corporal (ECC) entre os dois períodos avaliados. A média de ECC das fêmeas na avaliação feita entre 30 e 45 dias pós-parto foi inferior à media dessa variável na avaliação pré-parto $(\mathrm{P}<0,05)$. Essa é uma situação comum, mesmo em vacas de corte, que, embora não tenham grande produção leiteira, passam por um período de balanço energético negativo após o parto, que leva ao consumo das reservas corporais, influenciando no ECC (DISKIN et al., 2003).

Não houve diferenças entre a ocorrência de problemas pós-parto nos diferentes núcleos ou grupos de tratamento. Em todos os rebanhos e tratamentos a ocorrência de problemas foi baixa $(4,32 \%)$. FERNANDES et al. (2002b) relatam uma menor incidência de infecção uterina em vacas leiteiras tratadas com cloprostenol sódico em relação a animais não tratados. Essa diferença provavelmente ocorreu pela maior incidência desse tipo de patologia em gado leiteiro. Segundo a literatura, existe maior ocorrência significativa em gado leiteiro: 32,3\% (MILLER \& DORN, 1990),
25,3\% (LOEFFLER et al., 1999), 18,8\% (FERNANDES et al., 2000).

Não foram encontradas diferenças $(\mathrm{P}>0,05)$ entre o desempenho reprodutivo (nenhuma das variáveis analisadas) entre todos os animais nos diferentes núcleos nem entre um mesmo grupo de tratamento entre os núcleos de produção avaliados (Tabelas 2 a 4). Diante dessa situação, a análise dos tratamentos foi feita em conjunto com os animais desses três locais.

$\mathrm{O}$ efeito dos diferentes tratamentos foi semelhante nos três núcleos. A diferença de desempenho dos animais tratados em relação ao controle mostra a repetibilidade dos tratamentos. FERNANDES et al. (2005), trabalhando também com rebanhos de corte, relataram resultados semelhantes, confirmando que a utilização do cloprostenol no pós-parto imediato pode melhorar a eficiência reprodutiva.

Em gado leiteiro, segundo FERNANDES et al. (2002ab), os efeitos na melhoria da performance reprodutiva dos animais tratados foram maiores dos que os apresentados neste trabalho. Isso provavelmente pode ter ocorrido pelas diferenças de manejo e genética entre os rebanhos de corte e leite. No caso do gado de corte, a presença de bezerro junto das vacas (ao pé da vaca), durante todo o período pós-parto, pode influenciar o retorno à atividade ovariana (BUTLER, 2005). Outros trabalhos com gado de corte (FERNANDES et al., 2005) mostraram efeitos muito semelhantes aos encontrados no presente estudo (Tabela 2).

Tabela 2: Média geral de intervalo parto-primeira inseminação e nos diferentes núcleos de produção, nos diferentes grupos de tratamento

\begin{tabular}{llllll}
\hline Tratamento & & Média & \multicolumn{3}{l}{ Núcleos } \\
\cline { 3 - 6 } & $\mathrm{N}$ & Geral & 1 & 2 & 3 \\
\hline Controle & 152 & $88,77 \pm 23,64^{\mathrm{a}}$ & $92,16 \pm 25,35^{\mathrm{a} 1}$ & $85,66 \pm 28,90^{\mathrm{a} 1}$ & $90,40 \pm 26,84^{\mathrm{a} 1}$ \\
$0,530 \mathrm{mg}$ & 151 & $77,59 \pm 26,95^{\mathrm{b}}$ & $81,52 \pm 26,07^{\mathrm{b} 1}$ & $75,78 \pm 27,12^{\mathrm{b} 1}$ & $78,30 \pm 26,61^{\mathrm{b} 1}$ \\
$1,060 \mathrm{mg}$ & 158 & $76,22 \pm 26,28^{\mathrm{b}}$ & $80,55 \pm 24,75^{\mathrm{b} 1}$ & $74,32 \pm 23,33^{\mathrm{b} 1}$ & $75,60 \pm 31,25^{\mathrm{b} 1}$ \\
\hline Média Núcleo & & & $85,11 \pm 31,35^{1}$ & $78,61 \pm 28,46^{1}$ & $82,95 \pm 29,54^{1}$ \\
\hline
\end{tabular}

Médias seguidas pela mesma letra na coluna não diferem a $5 \%$ de probabilidade.

Médias seguidas pelo mesmo número na linha não diferem a 5\% de probabilidade.

Segundo SHELDON et al. (2000), existe correlação positiva entre a involução uterina e o retorno à atividade ovariana pós-parto. Esses autores afirmam que o retardo na involução uterina pode atrasar a presença de atividade funcional nos ovários, retardando o início do primeiro cio pós-parto. Embora não se tenha avaliado o grau de involução uterina no trabalho em questão, o mecanismo descrito acima provavelmente ocorreu, pois os animais tratados com cloprostenol sódico tiveram involução uterina acelerada, possibilitando retorno mais rápido à atividade reprodutiva pós-parto. Esses achados corroboram os resultados de ALBUQUERQUE et al. (1997) que, embora trabalhando com aplicação do mesmo análogo sintético em outro período pós-parto (12 a 18 dias), relataram resultados semelhantes, ou seja, involução uterina mais rápida naqueles animais que receberam 
o tratamento. HENDRICKS et al. (2005), utilizando análogos da PGF2 $\alpha$ num período pós-parto mais avançado, não conseguiram benefícios em relação à eficiência reprodutiva. Nesse mesmo período, três a cinco semanas após o parto, HIRSBRUNNER et al. (2006), utilizando apenas uma dose de PGF2 $\alpha$, também não viram efeitos benéficos em vacas leiteiras. É difícil comparar resultados de diferentes trabalhos realizados com animais e em condições distintas, visto a gama de variáveis relacionadas.

Segundo MARQUES JÚNIOR (1993), a prostaglandina2 $\alpha$ é uma das substâncias mais importantes na involução uterina da vaca. Os análogos sintéticos, no caso o cloprostenol, podem atuar de forma semelhante, de forma benéfica, nesse processo. Situação semelhante foi por FERNANDES et al. (2001) para vacas com retenção de placenta que apresentaram retardo na involução uterina e no retorno à atividade ovariana pós-parto.

Não foram observadas diferenças em relação ao número de serviços por concepção nos diferentes grupos e rebanhos avaliados (Tabela 3 ).

Tabela 3: Média geral de número de serviços por concepção e nos diferentes núcleos de produção, nos diferentes grupos de tratamento

\begin{tabular}{llllll}
\hline Tratamento & & Média & \multicolumn{3}{c}{ Núcleos } \\
\cline { 3 - 5 } & $\mathrm{N}$ & Tratamento & 1 & 2 & 3 \\
\hline Controle & 152 & $1,41 \pm 0,62^{\mathrm{a}}$ & $1,40 \pm 0,62^{\mathrm{a} 1}$ & $1,42 \pm 0,62^{\mathrm{a} 1}$ & $1,41 \pm 0,59^{\mathrm{a} 1}$ \\
$0,530 \mathrm{mg}$ & 151 & $1,36 \pm 0,54^{\mathrm{a}}$ & $1,37 \pm 0,67^{\mathrm{a}}$ & $1,37 \pm 0,49^{\mathrm{a} 1}$ & $1,35 \pm 0,64^{\mathrm{a} 1}$ \\
$1,060 \mathrm{mg}$ & 158 & $1,38 \pm 0,70^{\mathrm{a}}$ & $1,37 \pm 0,72^{\mathrm{a} 1}$ & $1,39 \pm 0,77^{\mathrm{a} 1}$ & $1,39 \pm 0,71^{\mathrm{a} 1}$ \\
\hline Média Núcleo & & & $1,39 \pm 0,71^{1}$ & $1,41 \pm 0,58^{1}$ & $1,37 \pm 0,66^{1}$ \\
\hline
\end{tabular}

Médias seguidas pela mesma letra na coluna não diferem a $5 \%$ de probabilidade.

Médias seguidas pelo mesmo número na linha não diferem a $5 \%$ de probabilidade.

Também num estudo utilizando cloprostenol no pós-parto em gado de corte, FERNANDES et al. (2005) não encontraram diferenças entre os grupos tratados e controle em ralação ao número de serviços por concepção. Em gado leiteiro, porém, FERNANDES et al. (2002b) mostraram que essa variável foi menor nas vacas do grupo tratado com duas doses de cloprostenol. Essa diferença provavelmente pode ter ocorrido porque os animais neste trabalho apresentaram menor ocorrência de infecção uterina. As infecções uterinas podem deixar, por algum tempo, sequelas no endométrio que prejudicam a fertilidade (SHELDON et al., 2000).

Em decorrência do menor intervalo partoprimeiro cio, como não houve diferenças no número de serviços por concepção, os animais tratados apresentaram menor período de serviços $(\mathrm{p}<0,05-$ Tabela 4).
O tratamento com cloprostenol, independente da dose, foi eficiente em acelerar o retorno à atividade reprodutiva pós-parto e também o intervalo do parto à concepção, denominado período de serviços. Os resultados (Tabela 4) mostram que, em média, os animais que receberam a dose de $0,530 \mathrm{mg}$ de cloprostenol ficaram gestantes 11 dias mais cedo que o grupo controle. Aqueles que receberam $1,060 \mathrm{mg}$ do mesmo produto ficaram gestantes cerca de 12,1 dias mais cedo; logo, não houve diferença ( $p>0.05)$ entre as doses utilizadas. Considerando um período de gestação médio de 290 dias em fêmeas zebuínas, para a produção de um bezerro/ano, o intervalo entre o parto e a próxima concepção deve ser no máximo de 75 dias (FERREIRA et al., 1999); portanto, a antecipação de 10 dias obtida no presente trabalho é uma ferramenta valiosa para aumentar a eficiência reprodutiva de gado de corte.

Tabela 4: Média geral de período de serviços e nos diferentes núcleos de produção, nos diferentes grupos de tratamento

\begin{tabular}{llllll}
\hline Tratamento & & Média & \multicolumn{3}{l}{ Núcleos } \\
\cline { 3 - 5 } & $\mathrm{N}$ & Tratamento & 1 & 2 & 3 \\
\hline Controle & 152 & $97,34 \pm 26,54^{\mathrm{a}}$ & $99,96 \pm 30,07^{\mathrm{a} 1}$ & $94,89 \pm 32,73^{\mathrm{a} 1}$ & $98,44 \pm 29,98^{\mathrm{a} 1}$ \\
$0,530 \mathrm{mg}$ & 151 & $86,38 \pm 28,81^{\mathrm{b}}$ & $89,79 \pm 29,73^{\mathrm{b} 1}$ & $83,77 \pm 31,11^{\mathrm{b} 1}$ & $86,69 \pm 28,69^{\mathrm{b} 1}$ \\
$1,060 \mathrm{mg}$ & 158 & $85,23 \pm 30,12^{\mathrm{b}}$ & $88,97 \pm 28,74^{\mathrm{b} 1}$ & $83,05 \pm 28,42^{\mathrm{b} 1}$ & $85,78 \pm 33,01^{\mathrm{b} 1}$ \\
\hline Média Núcleo & & & $92,98 \pm 32,12^{1}$ & $85,85 \pm 33,18^{1}$ & $90,34 \pm 32,19^{1}$ \\
\hline
\end{tabular}

Médias seguidas pela mesma letra na coluna não diferem a $5 \%$ de probabilidade.

Médias seguidas pelo mesmo número na linha não diferem a 5\% de probabilidade. 
Segundo FERNANDES et al. (2002ab), animais que receberam dose maior de cloprostenol $(1,060 \mathrm{mg})$, porém divididas em duas aplicações $(0,530+0,530 \mathrm{mg})$, apresentaram desempenho reprodutivo superior a uma única aplicação de $0,530 \mathrm{mg}$. Esse efeito provavelmente não foi observado no trabalho em questão, pois, como o cloprostenol possui meia-vida curta, a maior dose (1,060mg), numa única aplicação, pode ter saturado os receptores existentes e parte dela ter sido metabolizada sem promover qualquer efeito. Isso, porém é uma suposição que deve ser avaliada em trabalhos posteriores.

\section{CONCLUSÕES}

A aplicação de cloprostenol sódico nas duas doses utilizadas, no pós-parto imediato de vacas de corte, acelera o retorno à atividade reprodutiva $\mathrm{e} o$ período de serviços. Não há diferença no desempenho reprodutivo dos animais que receberam as diferentes doses de cloprostenol testadas, 0,530 ou $1,060 \mathrm{mg}$. Pelo menor custo, recomenda-se a dose de $0,530 \mathrm{mg}$.

\section{REFERÊNCIAS}

ALBUQUERQUE, F.T.; CHOW, L.A.; SÁ, W.F.; FERREIRA, A,M,; TRES, J.;E. JACOB, J.C.F. Efeito do cloprostenol (ICI-80.996) sobre a involução uterina em vacas mestiças Holandes-Zebu. Arquivo Brasileiro de Medicina Veterinária e Zootecnia, v.49, n.2, p.181-187, 1997.

BENCHARIF, D.; TAINTURIER, D.; SLAMA, H.; BRUYAS, J.F.; BATTUT, I.; FIENI, F. Prostaglandins and post-partum period in the cow. Revue de Medecine Veterinaire, v.151, n.5, p.401-408; 2000.

BUTLER, W.R. Inhibition of ovulation in the postpartum cow and the lactating sow. Livestock Production Science, v.34, p.18-27, 2005.

CHAGAS, W.N.; NOGUEIRA, L.A.G.; BENEVIDES FILHO, I.M. Avaliação histológica da involução uterina em vacas da raça Nelore. Revista Brasileira de Reprodução Animal, v.22, n.3, p.164-168, 1998.

DISKIN, M.G., MACKEY,D.R., ROCHE , J.F.,SREENAN, J.M. Effects of nutrition and metabolic status on circulating hormones and ovarian follicle development in cattle. Animal Reproduction Science, v.78, p. 345-370, 2003.

FERNANDES, C.A.C. Alternativas para tratamento de retenção de placenta em gado de leite. Revista Brasileira de Reprodução Animal, v.23, 442-444, 1999.

FERNANDES, C.A.C., VIANA, J.H.M., FERREIRA, A.
M.; Efeito do cloprostenol sódico no pós-parto de vacas leiteiras e retorno á atividade reprodutiva. A Hora Veterinária, v.126, p.13-17, 2002a.

FERNANDES, C.A.C., VIEIRA, M.R.; VALLE, M.A.G.; Efeito do cloprostenol sódico no pós-parto de vacas leiteiras sobre a incidência de infecções uterina e fertilidade futura. A Hora Veterinária, v.127, p.41-45, $2002 b$.

FERNANDES, C.A.C.; COSTA, D.S.;VIANA, J.H.M. Impacto da retenção de placenta sobre a performance reprodutiva de vacas leiteiras. Revista Brasileira de Reprodução Animal, v.25, n.1, p.26-30, 2001.

FERNANDES, C.A.C.; FIGUEIREDO, A.C.S. Avanços na utilização de prostaglandinas na reprodução de bovinos. Revista Brasileira de Reprodução Animal, v.31, n.3, p.406-414, 2007.

FERNANDES, C.A.C.; GRANJA Jr, P.F.; CANELLA FILHO, C.; CASTRO, D.A.M.; FERREIRA, A.M. Eficiência da Florfenicol para tratamento sistêmico de infecções uterinas agudas em bovinos. A Hora Veterinária, v.118, p.112-115, 2000.

FERNANDES, C.A.C.; OLIVEIRA, E.R.; VASCONCELOS, T.D. Efeitos do cloprostenol sódico no pós-parto de vacas de corte. In: XVI CONGRESSO BRASILEIRO DE REPRODUÇÃO ANIMAL, 2005, Goiânia-GO. Anais... 2005. v. 1, p. 351-351

FERREIRA, A. M.; TORRES, C. A. A.; SÁ, W. F.; VIANA, J. H. M. . Consumo e custo da alimentação para recuperação da atividade ovariana luteal cíclica de vacas mestiças leiteiras com anestro. Pesquisa Agropecuária Brasileira, v. 34, n.1, p. 129-133, 1999.

FIGUEIREDO, M.M.N.; FONSECA, F.A.; TORRES, C.A.A.; GALIMBERTI, A.M.; ALMEIDA, C.D. Dinâmica folicular ovariana de vacas leiteiras no pósparto após tratamentos com Buserelina (GnRH) e Cloprostenol (PGF2 $\alpha$ ). Revista Brasileira de Zootecnia, v.29, n.3, p.89-92, 2000.

HENDRICKS K.E.M., BARTOLOME J.A., MELENDEZ, P., RISCO, C., ARCHBALD, L.F. Effect of repeated administration of PGF2a in the early post partum period on the prevalence of clinical endometritis and probability of pregnancy at first insemination in lactating dairy cows. Theriogenology, v. 63, p.67-81, 2005.

HIRSBRUNNER, G.; BURKHARDT, H.W.; STEINER, A. Effects of a single administration of prostaglandin F2alpha,or a combination of prostaglandin F2alpha and prostaglandin E2,or placebo on fertility variables in dairy cows $3-5$ weeks post partum, a randomized, double-blind clinical trial. Reproduction Biology and Endocrinology, v. 4, p.34-42, 2006.

Kask K, Kindahl H, Gustafsson H,. Bacteriological and histological investigation of the postpartum bovine uterus in two Estonian dairv herds. Acta Veterinaria 
Scandinavica, v.39, p.423-432, 1998.

LOEFFLER, S.H.; SCHUKKEN, Y.H.; DE VRIES, M.J. The effects of time of disease occurrence, milk yield, and body condition on fertility of dairy cows. Journal of Dairy Science, v.82, n.12, p.2589-2604, 1999.

MARQUES JUNIOR, A. P.;BARRETO FILHO, J. B.;NASCIMENTO, E. F.;R.L.. Alguns aspectos histológicos do placentomo da vaca zebu (Bos taurus indicus) no posparto. Revista Brasileira de Reproducao Animal, v. 19, n.34, p. 161-164, 1995.

MILLER, G.Y.; DORN, C.R. Costs of dairy cattle diseases to producers in Ohio. Preventive Veterinary Medicine, v.8, n.2-3, p.171-182, 1990.

SHELDON, I.M.; NOAKES, D.E.; DOBSON, H. The influence of ovarian activity and uterine involution determined by ultrasonography on subsequent reproductive performance of dairy cows. Theriogenology, v.54, n.3, p.409-419, 2000.

TANIKAWA M., ACOSTA, T.J.. FUKUI, T., MURAKAMIA, S., KORZEKWA, A. Regulation of prostaglandin synthesis by interleukin-1 in bovine endometrium during the estrous cycle. Prostaglandins \& other Lipid Mediators, v. 78, p. 279-290, 2005.

ZAIEM, I.; TAINTURIER, D.; OTHMEN, H.B.; BECKERS, J.F.; CHEMLI, J. Retained placenta and infertility: etiproston treatment and pregnancy associated glucoprotein. Revue de Medecine Veterinaire, v.148, n.8-9, p.725-732; 1997.

ZANCHET, E. Efeito de duas injeções de prostaglandina F2 $\alpha$ após o parto na performance reprodutiva de vacas leiteiras e eficiência reprodutiva entre raças Holandesa e Jersey. A Hora Veterinária, ano 24, n. 143, p.32-35, 2005. 